\title{
Impact of Dielectric Changes on L-Band 3-D SAR Reflectivity Profiles of Forest Volumes
}

\author{
Matteo Pardini, Member, IEEE, Konstantinos Papathanassiou, Fellow, IEEE, and Fabrizio \\ Lombardini, Senior Member, IEEE
}

\begin{abstract}
Synthetic Aperture Radar (SAR) Tomography (TomoSAR) allows the reconstruction of the vertical distribution of the power backscattered by natural volumes by combining multiple SAR images acquired with slightly different incidence angles. Being a "radar" quantity, the profile depends on the radar frequency, polarization, and acquisition geometry, the 3-D distribution of the scattering elements and their dielectric properties. The characterization of each one of these factors is crucial to enable the extraction of physical 3-D structure attributes from TomoSAR profiles.

The objective of this work is to investigate how the vertical distribution of the backscattered power at L-band is affected by seasonal and weather-induced changes. Radiometric (e.g. ground and volume powers) and geometric (e.g. center of mass of the volume-only profiles and phase centers of the volume scattering layers) parameters have been estimated under different weather and season conditions and compared. Then, TomoSAR data sets affected by dielectric non-stationarity (i.e. variability) have been considered in order to assess the invariance degree of each radiometric and geometric parameter. This analysis has been carried out by processing four L-band airborne TomoSAR data sets acquired before and after a rainfall and in spring and autumn over the Traunstein forest (South of Germany).
\end{abstract}

Index Terms-Synthetic aperture radar (SAR), SAR tomography (TomoSAR), dielectric changes, forest structure.

\section{INTRODUCTION}

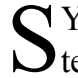
YNTHETIC Aperture Radar Tomography (TomoSAR) techniques enable the reconstruction of the vertical distribution of the backscattered power, known also as reflectivity profile, by combining multiple SAR images acquired with slightly different incidence angles (e.g., along slightly displaced tracks or orbits) [1]. This has been demonstrated extensively at different radar frequencies and in particular for different forest types and conditions [2]-[6]. A reflectivity profile is a "radar" quantity, and depends on the radar frequency and polarization, the acquisition geometry, the 3-D distribution of the scatterers in space, and their dielectric properties. Concerning the latter factor and fixed all the others,

This work was supported by the Helmholtz Alliance Remote Sensing and Earth System Dynamics funded by the Initiative and Networking Fund of the Helmholtz Association.

M. Pardini and K. Papathanassiou are with the German Aerospace Center (DLR), Microwaves and Radar Institute, 82234 Wessling, Germany.

F. Lombardini is with the Department of "Ingegneria dell'Informazione", University of Pisa, 56122, Pisa, Italy. it is a fact that radar backscatter variations from forests over time are mostly related to changes in the dielectric properties of the soil and the vegetation layer. Such changes have been observed in polarimetric and/or interferometric measurements of different forest types in ground-based, airborne and space borne SAR experiments at different frequencies [7]-[14].

The dielectric properties of the vegetation layer depend on its water status, intended as water content and surface moisture level. The water status varies from hour to seasonal scales, and depends tree species, tree structure (trunk, stem, branches), and environmental stress [15]. A variation of the water status is caused also by rain events [10]. Indeed, during a rain event, the forest canopy can retain up to a certain amount of water, above which the extra amount drops to the ground, increasing its moisture content. At the same time, the water content inside the leaves and branches increases as well due to changes in transpiration. Accordingly, the related wave absorption phenomena increase the electromagnetic attenuation of the canopy layers [10]. In general, the temporal extent and the intensity of these effects depend not only on the single trees, but also on their 3-D distribution in space. Seasonal changes of water status are instead more difficult to be characterized even at a single tree level. The water content in the trunk generally has a peak in the autumn and winter months (minimum transpiration), and can decrease strongly through spring and summer (maximum transpiration). However, different species exhibit different trends and timings [15].

The objective of this paper is to characterize how weatherinduced and seasonal dielectric variations affect TomoSAR Lband reflectivity profiles of a temperate forest site. The intention is not to interpret profile changes, but to assess them qualitatively and quantitatively. In particular, profile parameters that are more sensitive to dielectric changes have been identified. Four different airborne TomoSAR data sets acquired over the temperate forest of Traunstein located in the southern part of Germany have been processed. They are described in Section II. Out of the four data sets, two have been acquired before and after a rainfall event, and the other two in different seasons (spring and autumn). L-band waves penetrate through the canopy and interact with the underlying ground. In order to investigate ground and volume changes, their backscattered signals need to be separated. Their total backscattered power has been estimated and variations quantified. Then, volume-only reflectivity profiles have been 
estimated and related parameters extracted, such as the profile center of mass (that at first order is equivalent to an interferometric phase center) and both the position and relative powers of a set of scattering layers along height. The used algorithms are described in Section III, and the real data results are reported in Section IV. Then, the same analysis has been repeated on data sets in non-stationary dielectric conditions "synthesized" from the available stationary ones (Section V). In this way, the effects of dielectric nonstationarities during the acquisition time span on the reconstructed profile could be assessed. The conclusions of the analysis are reported in Section VI, together with a discussion of the implications of dielectric non-stationarities on the reconstruction of the reflectivity profiles and on TomoSAR implementations from space borne SAR platforms.

\section{Test Site And Data Sets}

\section{A. The Traunstein forest}

The "Traunstein Bürgerwald" is a highly structured, mixed, temperate forest located close to the city of Traunstein, South of Germany $\left(47.857742^{\circ}\right.$ E, $\left.12.657801^{\circ} \mathrm{N}\right)$. An optical ortophoto of the forest is shown in Fig. 1(a). The forest is predominantly composed of Norway spruce (Picea abies), European silver fir (Abies alba), European beech (Fagus sylvatica) and Sycamore maple (Acer pseudoplatanus). Through the years, the Traunstein forest is being reconverted from a homogeneous one-age forest to a structurally rich, heterogeneous forest. Most forest stands are complex in terms of tree species richness and heterogeneous stand structures due to "close-to-nature" silviculture, which is reflected in a broad distribution of growth stages. The topography ranges from 630 to $720 \mathrm{~m}$ above sea level and includes small areas with steep slopes. Forest top heights range from 10 up to $40 \mathrm{~m}$. The mean biomass level is about $200 \mathrm{Mg} / \mathrm{ha}$ and is significantly higher than other managed forests in the same ecological zone.

For the purpose of this study, the thematic map shown in Fig. 1(b) of the different growth stages is used as a reference. Using ground inventory data, forest management information, and optical high resolution images [16], the forest stands were classified into five different growth stages, namely "young", "growing", "mature", "transition" and "plenter". Young stands are constituted predominantly by young trees with low density. Density increases in the growing forest stands, which include some scattered taller trees above the young shorter ones. In mature stands, trees are homogeneously distributed, with a dense top vegetation layer. Stands in a transition stage are characterized by a regrowth of shorter trees below an older, taller and denser vegetation layer. Finally, stands in the "plenter" area are very heterogeneous in height and structure as a result of forest management practices. Structural complexity increases from the young to the "plenter" stands, with the exception of mature stands. Despite being old stands, they appear very homogenous. Within the five different stages, the classification of areas with a majority of broadleaved or coniferous trees is also available.

\section{B. Data sets}

Given its spatial heterogeneity and structure richness, the Traunstein forest has been established as a long-term monitoring site. Polarimetric, interferometric and tomographic acquisitions have been carried out since 2003 with the E-SAR and F-SAR airborne platforms of the German Aerospace Center (DLR), with the objective of not only to develop and validate forest parameter estimation algorithms, but also to

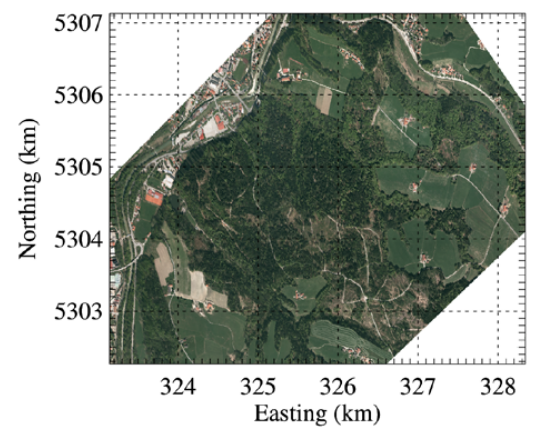

(a)

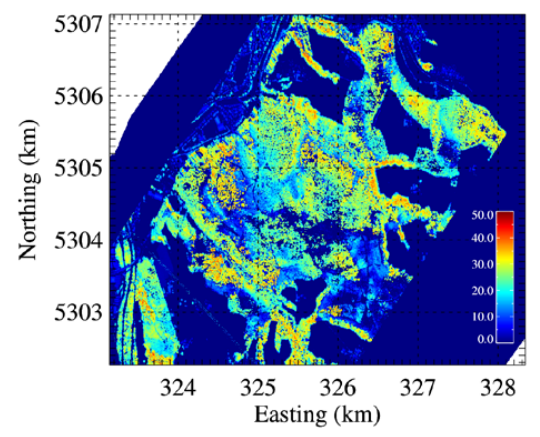

(c)

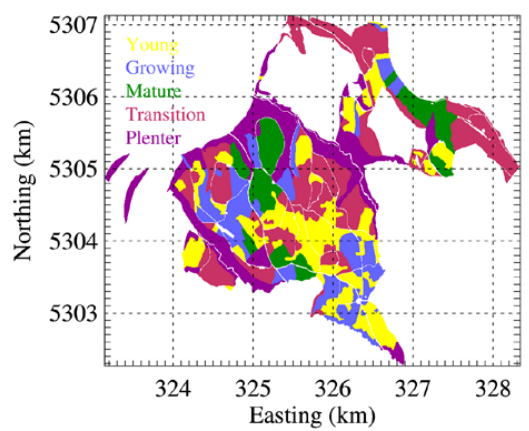

(b)

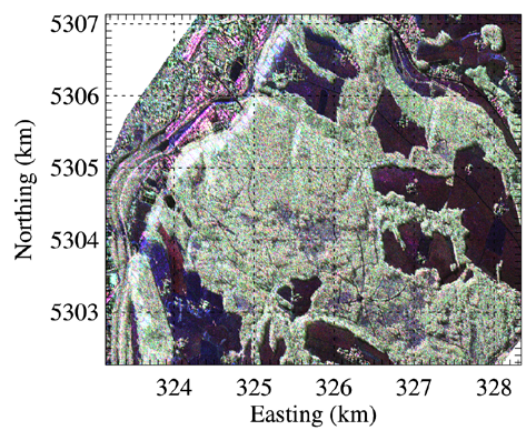

(d)

Fig. 1. Traunstein test site (South of Germany, UTM zone 33): (a) optical image; (b) map of growth stages; (c) lidar top height; (d) E-SAR L-band Pauli RGB composite image (acquisition of June 10, 2008). 
demonstrate their potential to document forest change [17], [18]. For validation purposes, airborne lidar and inventory measurements are systematically performed. Fig. 1(c) shows the lidar top tree height (first return) from an acquisition in September 2008.

In the same year 2008, L-band fully polarimetric TomoSAR data sets were acquired on June 7, 10, 12, 19 and 20. Each TomoSAR data set is composed by 5 tracks acquired in less than 1 hour, and the slave tracks had been designed to be nominally displaced in the horizontal direction by $5,10,15$, and $25 \mathrm{~m}$ with respect to the master track at a flight height of around $10000 \mathrm{ft}$ (equivalent to $3048 \mathrm{~m}$ ) above ground. A Pauli RGB composite image for the area of interest is shown in Fig. 1(d). While the acquisition on June 10 was carried out after a few days without precipitations, a significant rain event took place between June 10 and 12, as reported by the local weather stations of Nilling and Schönharting [17]. Therefore, these two data sets have been used in this paper to evaluate the impact of dielectric changes due to rainfall on the vertical reflectivity profiles. For brevity, these two data sets are referred as "dry" (June 10) and "wet" (June 12) in the following. The wind speed during the acquisitions was rather low (lower than $1 \mathrm{~m} / \mathrm{s}$ ), thus wind-induced temporal decorrelation can be neglected.

Fully polarimetric TomoSAR data were acquired also in spring (April 27, 28, and May 5, 11, 12) and autumn 2009 (October 27, 28, and November 5), flying at the same height as in 2008. The spring data sets consist of 7 tracks with horizontal displacements uniformly distributed within $30 \mathrm{~m}$. The autumn data sets consist of a variable number of uniformly distributed tracks with minimum and maximum displacement of $5 \mathrm{~m}$ and $15 \mathrm{~m}$. The data sets acquired on May 11 and October 28 have then been used to analyze seasonal vertical reflectivity changes. Both of them were acquired after rather dry days and in low wind conditions.

The expected tomographic performance in terms of vertical Rayleigh resolution and ambiguity-free height interval can be calculated from the maximum and minimum available vertical wavenumbers $k_{Z}$, respectively. In general, $k_{z}$ expresses the vertical sensitivity of the phase difference between two tracks, and it is calculated as [1], [19]:

$k_{Z}=\frac{4 \pi}{\lambda} \frac{\Delta \theta}{\sin \theta} \simeq \frac{4 \pi}{\lambda} \frac{B}{R \sin \theta \cos \theta}$,

where $\theta$ is the nominal incidence angle, $\lambda$ is the radar wavelength, $R$ is the slant-range distance, and $\Delta \theta$ is the incidence angle difference induced by the horizontal displacement $B$ between the two tracks. The vertical TomoSAR Rayleigh resolution is given by $\rho_{Z}=2 \pi / \max \left(k_{Z}\right)$ [1], while the ambiguity-free height interval is approximated as $\mathrm{HoA}=2 \pi / \min \left(k_{Z}\right)$ [1]. The $k_{Z}$ values of the acquisitions on June 10, 2008, are shown in Fig. 2. There are only small deviations with respect to the nominal values. Similar wavenumbers were obtained for the acquisition of June 12 . For these data sets, it results $\rho_{Z} \simeq 18 \mathrm{~m}$ and a $\mathrm{HoA} \simeq 90 \mathrm{~m}$ at the range coordinate in the middle of the area of interest. For

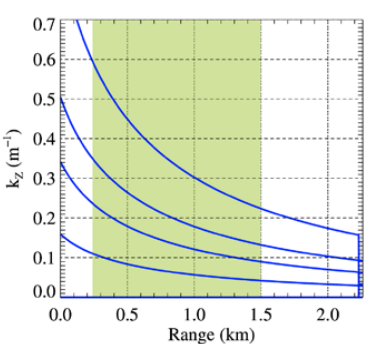

Fig. 2. Vertical wavenumbers of the five tracks realized during the acquisition on June 10, 2008 as a function of the range coordinate and averaged along azimuth. The range coordinate is relative to the (azimuthvariant) range at the processed swath start. The green box indicates the range extension of the area of interest, i.e. the one including the forest areas outlined in Fig. 1(b). A null vertical wavenumber is conventionally assigned to the master track.

TABLE I

SUMMARY OF E-SAR DATA PARAMETERS

\begin{tabular}{|c|c|c|}
\hline Campaign & TempoSAR 2008 & TempoSAR 2009 \\
\hline Frequency & \multicolumn{2}{|c|}{ L-band (wavelength $\sim 23 \mathrm{~cm}$ ) } \\
\hline Acquisition date & Jun. 10 / Jun. 12 & May 11 / Oct. 28 \\
\hline Season & Late spring & Spring / Autumn \\
\hline Weather conditions & Dry / Wet & Dry / Dry \\
\hline Wind speed & $<1 \mathrm{~m} / \mathrm{s}$ & $<1 \mathrm{~m} / \mathrm{s}$ \\
\hline $\begin{array}{c}\text { Resolution } \\
\text { Range } \times \text { azimuth, } \mathrm{m}\end{array}$ & \multicolumn{2}{|c|}{$2.12 \times 1.2$} \\
\hline $\begin{array}{l}\text { [min, max] incidence } \\
\text { angle, deg }(*)\end{array}$ & \multicolumn{2}{|c|}{$[32,45]$} \\
\hline Number of tracks & 5 & 4 \\
\hline $\begin{array}{c}\text { Max. vertical } \\
\text { wavenumber, rad } / \mathrm{m} \\
\text { (at mid range) }(*)\end{array}$ & 0.34 & 0.25 \\
\hline $\begin{array}{l}\text { Vert. resolution, } m \\
\text { (at mid range) }(*)\end{array}$ & $\sim 18$ & $\sim 25$ \\
\hline
\end{tabular}

the acquisition of October 28, 2009, the non-zero available $k_{Z}$ 's are very similar to the smallest 3 plotted in Fig. 2. Therefore, while the HoA does not change, $\rho_{Z}$ degrades to around $25 \mathrm{~m}$. However, this value is still small enough to allow two vertical resolution cells in the tallest stands. In order to obtain the same vertical resolution and the same number of tracks in the spring-autumn comparison, the TomoSAR analyses have been carried out only on a subset of the tracks in the spring data set (track displacements up to $15 \mathrm{~m}$ ). The main acquisition and data parameters for the considered sets are summarized in Table I.

\section{ALGORITHMS}

The TomoSAR data sets of 2008 and 2009 are composed by $K=5$ and $K=4$ images, respectively, and by $N_{P}=3$ polarimetric channels $(\mathrm{HH}, \mathrm{HV}, \mathrm{VV})$. After conventional focusing and interferometric processing, phase errors due to residual track positioning errors have been estimated and 
compensated for all data sets by using the tomographic autofocus concept [20], [21], adopting the method described in [21].

In the TomoSAR data model, the height-dependent phase differences for a height $z$ for each of the tracks are contained in the so-called steering vector $\mathbf{a}(z)$, with generic element $\{\mathbf{a}(z)\}_{k}=\exp \left(j k_{z, k} z\right), k=1, \ldots, K . k_{z, k}$ indicates here the vertical wavenumber associated to the $k$-th track with respect to the first (master) track. For a fixed range-azimuth coordinate, the TomoSAR information is completely represented by the covariance matrix $\mathbf{R}_{P}:=E\left\{\mathbf{y}_{P} \mathbf{y}_{P}^{H}\right\}$, where $\mathbf{y}_{P}$ is the $N_{P} K$-dimensional data vector obtained by stacking the $N_{P}$ single-polarimetric channels of the $K$ images one on top of the other, and $(\cdot)^{H}$ denotes the Hermitian operator. For each data set, $\mathbf{R}_{P}$ has been estimated on a $15 \mathrm{~m}$ $\times 15 \mathrm{~m}$ (slant range-azimuth) multi-look cell, corresponding to approximately 85 independent looks.

All data sets have been processed by applying the following steps in a sequential order:

1. Data wavenumber interpolation: due to residual flight inaccuracies, the realized tracks slightly differ from the nominal ones. Different TomoSAR acquisitions will result into different track displacement patterns. Thus, for the same location on ground, data vectors with the real available $k_{Z}$ distributions have been interpolated to uniform ones (with same number of tracks) in order to minimize changes of quality of the TomoSAR estimates with changing data set, but also to better condition TomoSAR focusing and parameter estimation.

2. Separation of ground and volume scattering: this step is necessary to reduce as much as possible the ground scattering contribution. In this way, the volume-only interferometric coherences become available, enabling the analysis of the volume-only vertical reflectivity profiles, the estimation of the ground and volume scattering powers, and the ground-to-volume power ratios.

3. Estimation of volume-only vertical reflectivity parameters: in a first instance, the center of mass of the volume-only reflectivity profiles and normalized integrated profile differences have been estimated. To further quantify changes, the profiles have been modelled as the sum of (up to 2) scattering layers of which height positions, widths and relative powers have been estimated.

These processing steps have been detailed in the following subsections.

\section{A. Data wavenumber interpolation}

Let $\mathbf{a}_{I}(z)$ be the steering vector corresponding to the interpolated uniform tracks. As outlined in [22], the interpolator is designed as a matrix $\mathbf{H}$ that linearly transforms $\mathbf{a}(z)$ into $\mathbf{a}_{I}(z)$ with the minimum possible average error within a height interval of interest. The interpolator design criterion can be written as [22]:
$\mathbf{H}=\arg \min _{\mathbf{H}} \int_{z_{1}}^{z_{M}}\left\|\mathbf{a}_{I}(z)-\mathbf{H a}(z)\right\|^{2} d z$,

where $\left[z_{1}, z_{M}\right]$ is the height interval of interest that includes all the scattering components. The solution $\mathbf{H}$ can be calculated in closed form as in [23] including a regularization factor that increases the robustness in the presence of noise and (small) residual baseline errors [24]. Being $\mathbf{H}$ the same for all polarimetric channels, the interpolated polarimetric vector and covariance matrix are:

$\mathbf{y}_{P, I}=\left(\mathbf{I}_{N_{P}} \otimes \mathbf{H}\right) \mathbf{y}_{P}, \mathbf{R}_{P, I}=\left(\mathbf{I}_{N_{P}} \otimes \mathbf{H}\right) \mathbf{R}_{P}\left(\mathbf{I}_{N_{P}} \otimes \mathbf{H}\right)^{H}$,

where " $\otimes$ " is the Kronecker matrix product.

The maximum wavenumber of the interpolated 2008 data sets has been chosen as the common maximum of the individual available sets. The same has been done for the 2009 data sets. The height interval needed to compute the interpolation matrix has been chosen with $z_{1}=z_{D T M}-10 \mathrm{~m}$ and $z_{M}=z_{\text {top }}+10 \mathrm{~m}$, where $z_{D T M}$ and $z_{\text {top }}$ are the lidar DTM (Digital Terrain Model) and the top forest height, respectively. Although both quantities can be estimated from the radar data, the lidar ones have been used in order not to introduce an additional degree of uncertainty in the following analysis. It is important remarking that the tightness of the interpolator height interval to the one occupied by the scattering components minimizes the interpolation errors across the different data sets [22].

\section{B. Separation of ground and volume scattering}

Following [25], separating ground and volume means estimating their polarimetric covariances and the volume-only interferometric coherences. This has been implemented assuming the validity of the Random-Volume-over-Ground (RVoG) model [19], i.e. assuming that (i) the volume-only reflectivity profile is the same across all polarimetric channels up to a scaling factor, and that (ii) the ground reflectivity profile is a Dirac- $\delta$ function. Thus, $\mathbf{R}_{P, I}$ is modelled as:

$\mathbf{R}_{P, I}=\mathbf{C}_{G} \otimes\left[\mathbf{a}_{I}\left(z_{D T M}\right) \mathbf{a}_{I}^{H}\left(z_{D T M}\right)\right]+\mathbf{C}_{V} \otimes \boldsymbol{\Gamma}_{V}$,

where $\boldsymbol{\Gamma}_{V}$ is the volume interferometric coherence matrix, corresponding to the volume only (normalized) reflectivity profile and $\mathbf{C}_{G}$ and $\mathbf{C}_{V}$ are the ground and volume polarimetric covariance matrices, respectively. A unique $\boldsymbol{\Gamma}_{V}$ can be estimated by regularizing the Sum-of-KroneckerProduct decomposition. A detailed description and discussion of this separation methodology is reported in [25]. The estimation of $\mathbf{C}_{G}$ and $\mathbf{C}_{V}$ follows the estimation of $\boldsymbol{\Gamma}_{V}$ by applying a least-squares optimization. The diagonal elements of $\mathbf{C}_{G}$ and $\mathbf{C}_{V}$ are the separated ground and volume powers in the different polarimetric channels. From them, the HH, HV and $\mathrm{VV}$ ground-to-volume ratios $\left(\mu_{\mathrm{HH}}, \mu_{\mathrm{HV}}\right.$, and $\left.\mu_{V V}\right)$ are 
calculated as well. Finally, once $\mathbf{C}_{G}$ and $\mathbf{C}_{V}$ are estimated, the classical polarimetric contrast optimization can be used to find the polarimetric channel with the maximum and minimum ground-to-volume ratios $\mu_{\max }$ and $\mu_{\min }$ [26].

\section{Estimation of volume-only vertical reflectivity parameters}

The vertical location of the Center of Mass (CoM) of the volume-only reflectivity profiles is defined by:

$$
\text { CoM: }=\frac{\int_{z_{1}}^{z_{M}} P_{B F}(z) z d z}{\int_{z_{1}}^{z_{M}} P_{B F}(z) d z},
$$

with $z_{1}$ and $z_{M}$ defined in Section II.A. $P_{B F}(z)$ is the (Fourier-based) beamforming profile estimated from the volume-only coherences, that is calculated as [27]:

$$
P_{B F}(z)=\mathbf{a}_{I}^{H}(z) \boldsymbol{\Gamma}_{V} \mathbf{a}_{I}(z) / K .
$$

The beamforming spectral estimator has been chosen in order to preserve power linearity as much as possible. The CoM defined in (6) can be understood as the interferometric phase center that can be estimated by means of two tracks only, at least at the first order, neglecting the lack of phase triangularity due to the presence of volume scattering [13].

A way to express the difference between two profiles $P_{B F}^{(1)}(z)$ and $P_{B F}^{(2)}(z)$ is to quantify the Normalized Integrated profile Difference (NID), which is defined as the square root of the energy of the difference of two profiles, normalized to the energy of the reference one:

$$
\mathrm{NID}:=\sqrt{\frac{\int_{z_{1}}^{z_{M}}\left[P_{B F}^{(2)}(z)-P_{B F}^{(1)}(z)\right]^{2} d z}{\int_{z_{1}}^{z_{M}}\left[P_{B F}^{(1)}(z)\right]^{2} d z}},
$$

where $P_{B F}^{(1)}(z)$ is taken as the reference. Notice from (7) that, as the interferometric coherences are employed, the NID quantifies changes in the vertical distribution of powers, but is independent of changes of the total backscattered power.

Both CoM and NID parameters quantify profile changes by means of an integral operation along height. So doing, they transform 1-D functions (i.e. the profiles or profile differences) into scalars with a loss of information. In order to increase the detail of the analysis, the profiles have been expressed as the sum of elementary contributions, or layers, as:

$P_{B F}(z)=\sum_{n=1}^{N_{L}} \alpha_{n} P_{B F, n}(z)$,

where $N_{L}$ is the number of layers (model order), $\alpha_{n}$ is their relative weight, i.e. $\sum_{n=1}^{N_{L}} \alpha_{n}=1$, and $P_{B F, n}(z)$ is the beamforming profile of the modelled interferometric coherences of the layer. The corresponding "true" layer reflectivity profile has been modelled as Gaussian-shaped, with width (formally standard deviation) $w_{n}$ and phase center height (mean value) $z_{0 n}$. The estimation of $\left\{\alpha_{n}, w_{n}, z_{0 n}\right\}_{n=1}^{N_{L}}$ has been implemented here as:

$\left\{\alpha_{n}, w_{n}, z_{0 n}\right\}_{n=1}^{N_{L}}=\arg \min \int_{z_{1}}^{z_{M}}\left[P_{B F}(z)-\sum_{n=1}^{N_{L}} \alpha_{n} P_{B F, n}(z)\right]^{2} d z$.

Since the optimization functional in (9) is linear in $\left\{\alpha_{n}\right\}_{n=1}^{N_{L}}$, it can easily be concentrated as a function of the non-linear parameters $\left\{w_{n}, z_{0 n}\right\}_{n=1}^{N_{L}}$ only [28]. Notice that $N_{L}$ is unknown as well, and the higher $N_{L}$, the higher the dimensionality of the modelling and its inversion. For the data sets at hand, however, it is reasonable to test only two cases, i.e. $N_{L}=1$ and $N_{L}=2$. This is mainly imposed by the ratio between the forest top height and the vertical TomoSAR resolution $\rho_{Z}$. Also, the comparison of the height $z_{0 \max }$ of the maximum of $P_{B F}(z)$ and $z_{\mathrm{CoM}}$ determines both the model order and the parameter estimation procedure:

- If either $\left|z_{0 \max }-\mathrm{CoM}\right|<\rho_{Z} / 10$ or $z_{\text {top }} \leq 2 \rho_{Z}$ (i.e. low vertical resolution), then one (dominant) scattering contribution is assumed to be present, and $N_{L}=1$. Thus, it is set $z_{01}=z_{0 \max }$ [29], $\alpha_{1}=1$ by default, and $w_{1}$ is estimated with a 1-D exhaustive search using (9).

- On the contrary, if $\left|z_{0 \max }-\mathrm{CoM}\right| \geq \rho_{Z} / 10$ and $z_{\text {top }}>2 \rho_{Z}$, the hypothesis $N_{L}=2$ is tested. The two layer phase center heights are estimated by using the M-RELAX algorithm [27], while the layer widths are estimated with a 2-D exhaustive search using (9). Afterwards, the layer relative weights are obtained in a closed-form. However, as the modelling is unconstrained, it can happen either that one of the relative weights is negative, or that their sum is larger than 1 (when both are positive). In the latter case a $10 \%$ tolerance can be considered. Since these two cases are inconsistent, the hypothesis $N_{L}=2$ is discarded, and $N_{L}=1$ is retained.

It is worth remarking that the profile fitting approach used here is just one possibility to quantify changes of the profiles. At the level of this analysis, there is no claim that the estimated parameters are meaningful in terms of physical forest structure.

\section{Estimation of Reflectivity Profile Parameters: RESULTS}

In this Section, the change of the TomoSAR vertical reflectivity profiles induced by dielectric changes is reported.

In Fig. 3, TomoSAR profiles estimated on the same forest transect for a fixed azimuth and varying slant range (from 370 $\mathrm{m}$ to $1550 \mathrm{~m})$ are shown for the 4 data sets under consideration and for the HH, HV and VV channels. From near to far range (left to right), the transect crosses tall mature 

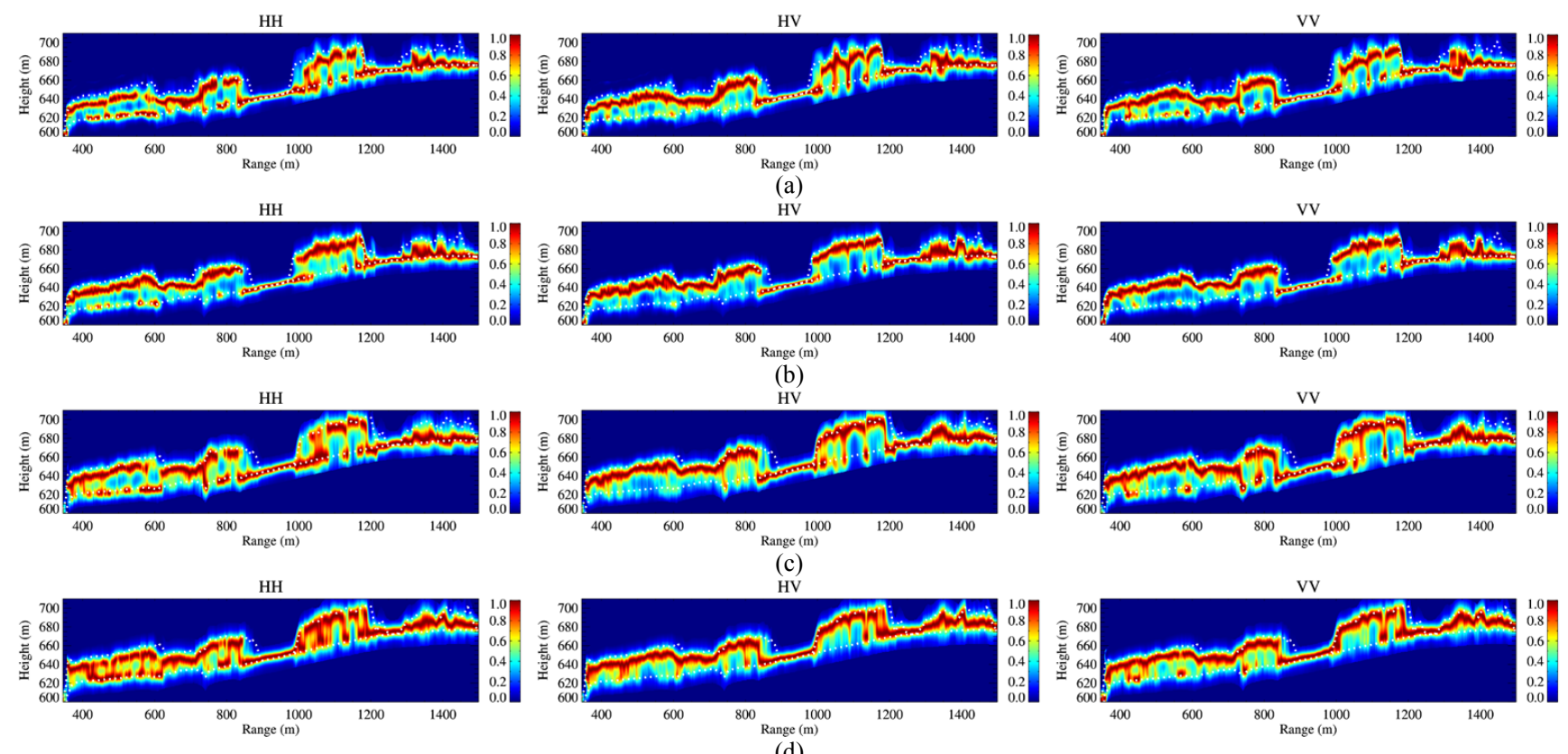

Fig. 3. TomoSAR profiles in the slant range - height plane of the same forest transect on: (a) June 10, 2008 (dry conditions); (b) June 12, 2008 (wet conditions); (c) May 11, 2009 (spring); (d) October 28, 2009 (autumn). Columns from left to right: HH channel, HV channel, VV channel. White dotted lines: lidar topography and top height.

stands (up to $35 \mathrm{~m}$ tall) until $850 \mathrm{~m}$ slant range, then a bare surface, and then transition and younger stands (around 30 and $15 \mathrm{~m}$ tall, respectively). For this analysis, the Capon spectral estimator [27], [30] has been used to improve vertical resolution. The employed multi-look degree reduces typical radiometric non-linearity issues [14]. Each profile has been normalized to its own maximum to emphasize the relative power levels among scattering components at different heights. Sidelobes (already reduced due to the interpolation to uniform tracks, and cleaned by Capon) and ambiguities (related to the HoA) out of the height interval of interest have been masked to ease interpretation. All profiles are well located between the lidar ground and top height. The estimated HV ground power is consistently lower than the $\mathrm{HH}$ and VV ground powers, while the $\mathrm{HH}$ one tends to be higher than the VV one, indicating the presence of dihedral contributions.

By comparing the "dry" and the "wet" profiles (first and second row in Fig. 3), it is apparent that in all polarizations the increased water on and (possibly) inside the vegetation elements decreases the ground power (relatively to the volume power) as a result of a stronger attenuation. A general change of the volume part of the profile can be observed as well. Due to the stronger attenuation, the maximum power contribution is mostly located close to the canopy top. For instance, volume parts that were relatively semi-transparent in dry conditions become well visible in wet conditions. An example can be found at $800 \mathrm{~m}$ slant range in $\mathrm{HH}$. Additionally, profiles with a homogeneous distribution of power along height in dry conditions exhibit a sharper distribution close to the canopy top in wet conditions, see e.g. $1000 \mathrm{~m}$ and $1200 \mathrm{~m}$ slant range. Changes in the young forest are more difficult to be seen due to the limited Rayleigh vertical resolution, however some effects along the lines of what described above are visible also thanks to the Capon super-resolution.

Seasonal changes are also apparent (third and fourth row of Fig. 3), despite the lower vertical resolution of the 2009 data sets with respect to the 2008 ones. Accounting for this loss of resolution, the spring profiles are judged to be comparable to the "dry" ones, as it is reasonable to expect given the similarity of external conditions. From spring to autumn, the change of water status in the forest volume results into a sensitive change of the profiles as well. In many stands, especially in $\mathrm{HH}$, the backscattered power components tend to distribute uniformly in height. This might be due to a redistribution of water content with a larger accumulation in the trunks [15]. At the same time, the profiles indicate a general increase of ground scattering in all polarizations.

\section{A. Changes of ground and volume powers}

A comparison of ground and volume powers estimated between dry and wet conditions, and spring and autumn, is reported in Fig. 4 for the HH channel. The other polarimetric channels exhibit essentially the same trends. From dry to wet conditions, the ground power mostly decreases in average by 1 to $2 \mathrm{~dB}$ for most of its range of variation [see Fig. 4(a) left], suggesting an increased volume attenuation. A significant ground power increase is however observed at the lower end of its range before the rainfall, i.e. below $-16 \mathrm{~dB}$. The most likely explanation is a bias in the estimation of the low ground power level [25]. In contrast, the volume power slightly increases [Fig. 4(a) right], consistently with what one would expect [10]. A global rise of volume power from spring to autumn is also found [Fig. 4(b) right]. As already observed from the normalized profiles of Fig. 3, the increased visibility of the ground corresponds to a general increase of its power 

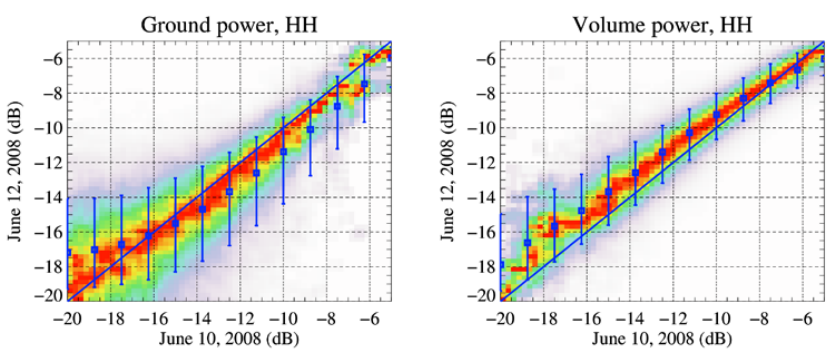

(a)
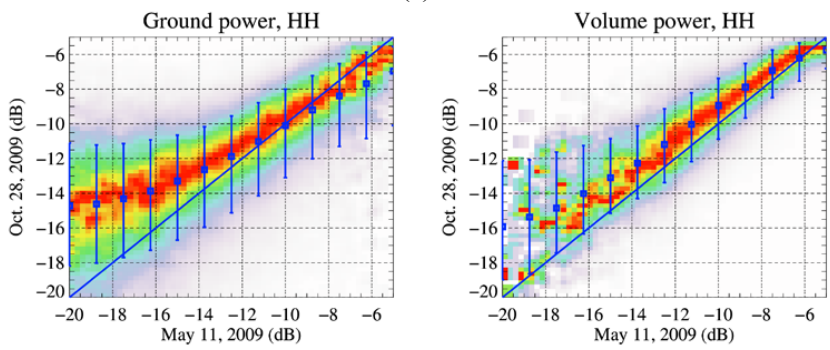

(b)

Fig. 4. Comparisons (2-D histograms) of the estimated ground (left column) and volume (right column) powers in the HH channel. (a) Wet against dry conditions; (b) autumn against spring. The square symbols indicate (conditioned) mean values, while the vertical bars delimit the interval where $75 \%$ of the estimates are found.

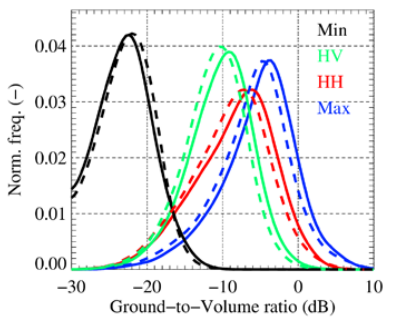

(a)

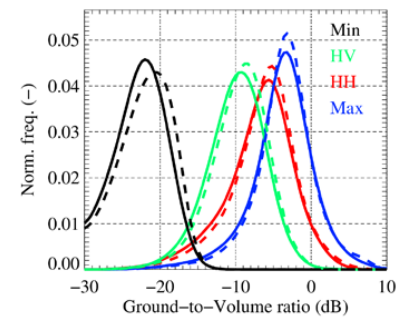

(b)
Fig. 5. Histograms of the estimated ground-to-volume ratios in the different polarimetric channels. (a) Dry (continuous) against wet (dashed) conditions; (b) spring (continuous) against autumn (dashed).

[Fig. 4 left]. The mean values of larger ground powers in spring remain almost unaltered in autumn.

The ground-to-volume ratios $\mu_{\mathrm{HH}}, \mu_{\mathrm{HV}}, \mu_{\min }$ and $\mu_{\max }$ have been calculated, and their histograms have been plotted in Fig. 5 for all data sets. Apparently, polarimetry increases the ground-to-volume ratio from $\mu_{\min }$ to $\mu_{\max }$ by up to $20 \mathrm{~dB}$ independently of the dielectric conditions. Furthermore, $\mu_{\max }$ is $3 \mathrm{~dB}$ larger than $\mu_{\mathrm{HH}}$ and around $8 \mathrm{~dB}$ larger than $\mu_{\mathrm{HV}}$.

The spatial distribution of $\mu_{H H}$ is shown in the maps of Fig. 6 for all data sets. $\mu_{H H}$ is in average lower in the most structurally heterogeneous and dense stands (mature, transition, "plenter") than in the less complex and sparser ones (young, growing). For each forest type, the change of $\mu_{H H}$ from dry to wet conditions is quantified in Fig. 7. The maps in Fig. 6(a) indicate a decrease of $\mu_{H H}$ especially in the mature, transition, and "plenter" stands. In particular, in the mature and transition stands $\mu_{H H}$ decreases from dry to wet conditions in more than $70 \%$ of the cells. Interestingly, mature stands with majority of broadleaved trees exhibit over larger
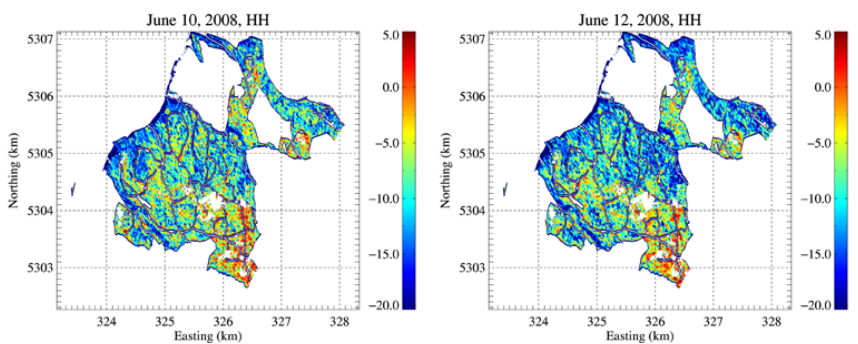

(a)
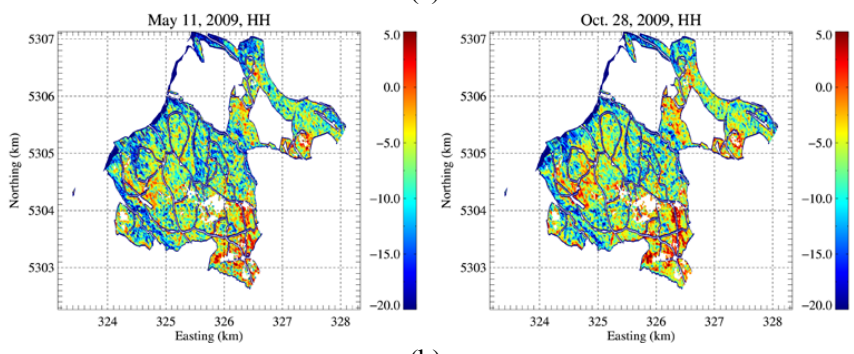

(b)

Fig. 6. Maps (UTM coordinates) of the estimated ground-to-volume ratios in the HH channel (dB). (a) Dry (left) against wet (right) conditions; (b) spring (left) against autumn (right).

areas $(80 \%$ of cells) a negative change, almost uniformly distributed between 0 and $-3 \mathrm{~dB}$. Conifers have a slightly narrower distribution with generally smaller changes. This species-driven difference might be related to a difference in water retention on the tree top caused by the presence of broad leaves. All stands are characterized by an increase of $\mu_{H H}$. However, increases larger than $3 \mathrm{~dB}$ are found only for $10 \%$ of the cases.

The maps of Fig. 6(b) show that seasonal changes of $\mu_{H H}$ tend to be more uniformly distributed in average between 1 and $2 \mathrm{~dB}$ across all stand types. As quantified in Fig. 8, $\mu_{H H}$ increases in more than $60 \%$ of the cells. In general, the histograms of Fig. 8 confirm that the distribution of the change is independent of forest type and dominant species.

All trends described above have also been observed in the other polarization channels, although they are more apparent in HH. As a final remark, it is worth noting that the map of May 11, 2009, reports larger values of $\mu_{H H}$ than the map of June 10, 2008, although both of them are in similar dry conditions. A reason for this may be the limited vertical resolution in 2009: in [25], it has been assessed that a degradation of the vertical resolution can cause an estimation bias up to $30 \%$. Of course, an additional intra-seasonal change cannot be excluded a priori.

\section{B. Changes of profiles: CoM and NID}

The maps of the estimated volume-only CoM, assumed common across the polarimetric channels as the reflectivity profile (see Section III.B), are shown in Fig. 9. In all the considered data sets, the CoM is higher for larger top heights, and reaches a maximum of $25 \mathrm{~m}$. However, it varies weakly across stands and types. Its variation from dry to wet conditions and from spring to autumn are quantified in the 2-D histograms of Fig. 10. It is apparent that the CoM generally increases from dry to wet conditions as a result of the noted increased attenuation. On the other hand, the CoM decreases 

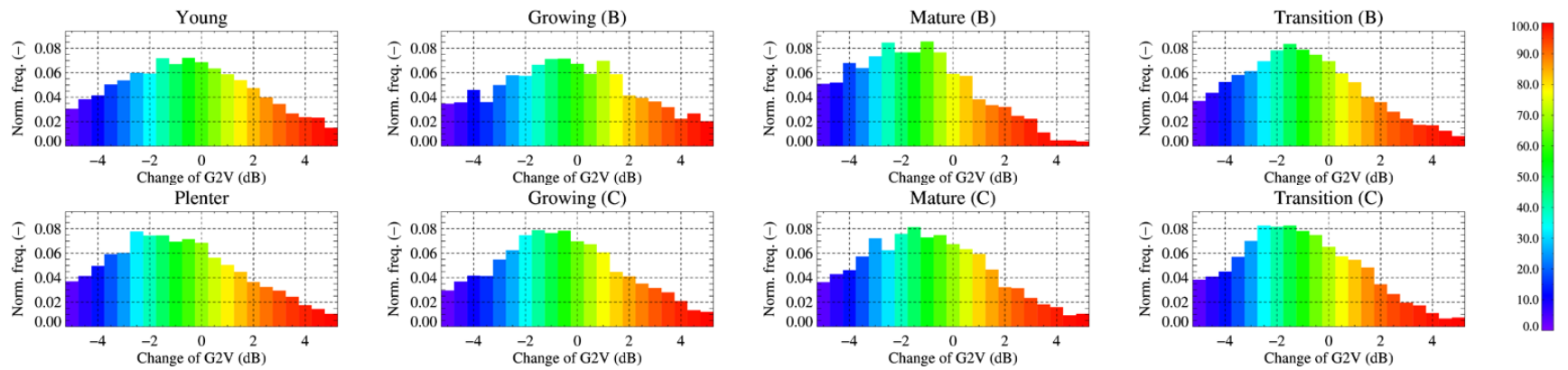

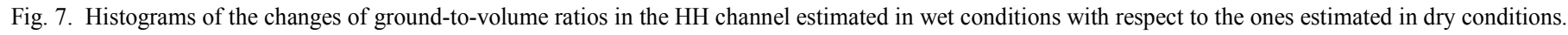
The different color represent the cumulated normalized frequency (\%); (B) stands for "broadleaved" and (C) stands for "conifers".
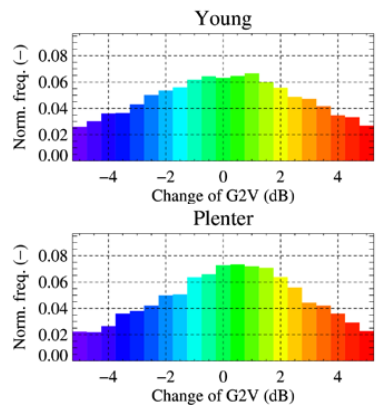

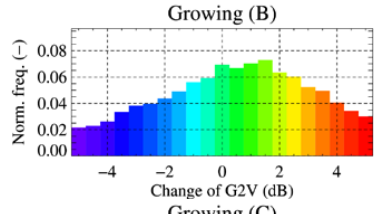
Growing (C)

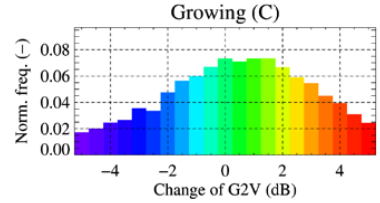

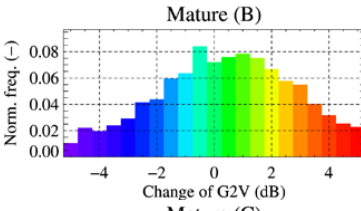
Mature (C)

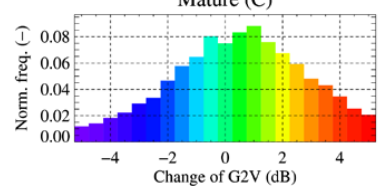

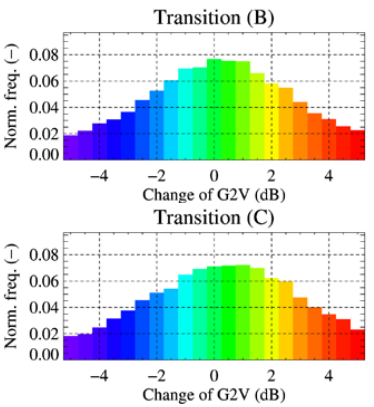

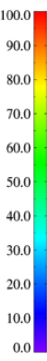

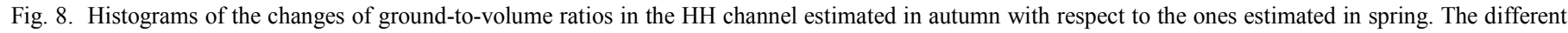
color represents the cumulated normalized frequency (\%); (B) stands for "broadleaved" and (C) stands for "conifers".

from spring to autumn, due to a higher relevance of volume scattering components located closer to the ground. Nevertheless, in both cases the average variation is in general within $20 \%$.

The NID, that is polarimetry-independent as the CoM, has been calculated for the "wet" and autumn data sets with respect to the "dry" and spring ones. The resulting maps and related histograms are shown in Fig. 11. In both cases, larger NID's occur in the more structurally complex areas, with a relevant average value around $40 \%$. However, the appearance of larger NID's is more frequent for seasonal changes, occurring also in younger stands. Interestingly, the NID seems not to be affected by the degradation of the vertical resolution neither from near to far range (South-East direction), nor from the 2008 to the 2009 data set. Furthermore, it is worth mentioning that very similar NID maps have been obtained with the higher resolution Capon spectral estimator in place of the Fourier-based beamforming.

\section{Changes of layer parameters}

The decomposition of the volume scattering into elementary layers presented in Section III.C has been applied to the volume-only profiles by testing the presence of $N_{L}=1$ or $N_{L}=2$ scattering layers. For all the data sets, the model order has been determined, and the layer phase center heights, widths, and relative weights have been estimated accordingly.

Fig. 12 shows the estimated $N_{L}$. In the "dry" and "wet" data sets [Fig. 12(a)], $N_{L}=2$ has been obtained for all areas with a more complex vertical structure, including also a part of stands in the growing stage. Almost all young stands provide $N_{L}=1$. Interestingly, there are no differences between the
$N_{L}$ maps in dry and wet conditions for more than $85 \%$ of cells. A different situation is shown by the spring and autumn maps [Fig. 12(b)]. The worse vertical resolution allows to reliably separate two layers only for a low(er) number of cells in areas with a larger structural complexity. By changing season, the spatial patterns in the $N_{L}$ maps are substantially preserved, with $75 \%$ of the cells remaining in the same $N_{L}$ class.

The fitted profile parameters are compared in Fig. 13, considering the cells with same order. For $N_{L}=1$, only the
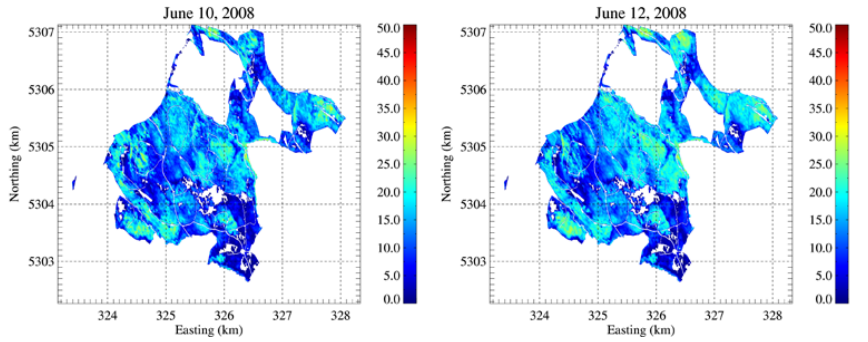

(a)
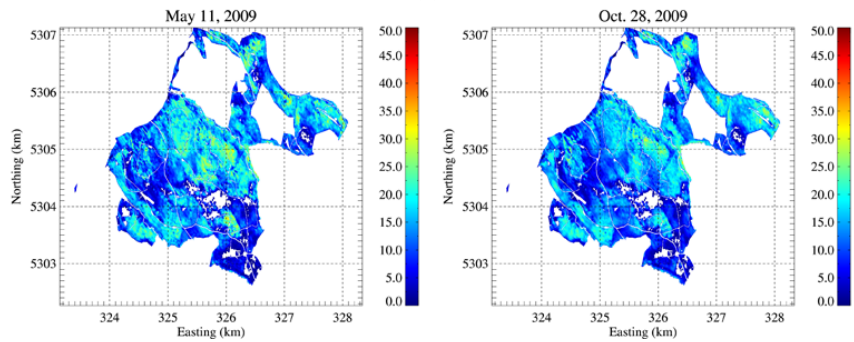

(b)

Fig. 9. Maps (UTM coordinates) of the estimated CoM (m) of the volumeonly profiles. (a) Dry (left) against wet (right) conditions; (b) spring (left) against autumn (right). 


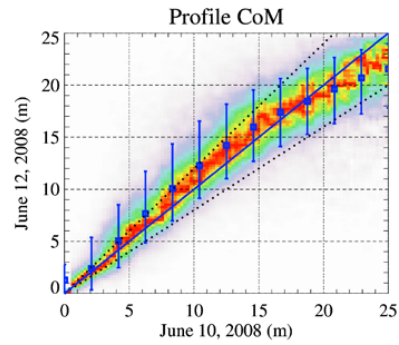

(a)

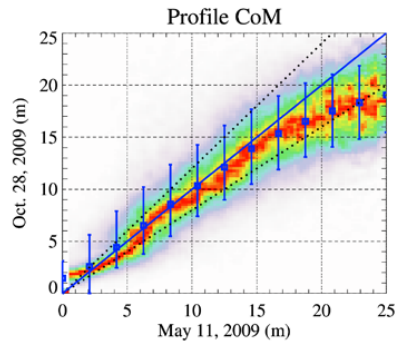

(b)
Fig. 10. Comparisons (2-D histograms) of the CoM (m) of the volume-only profiles. (a) Wet against dry conditions; (b) autumn against spring. The square symbols indicate (conditioned) mean values, while the vertical bars delimit the interval where $75 \%$ of the estimates are found. The dotted lines delimit the $20 \%$ variation interval.
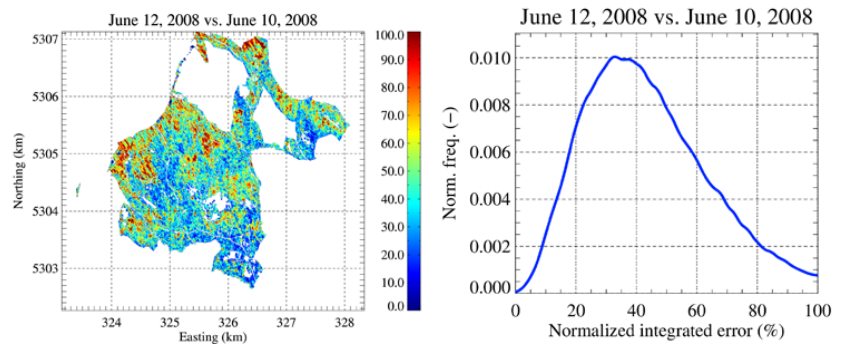

(a)
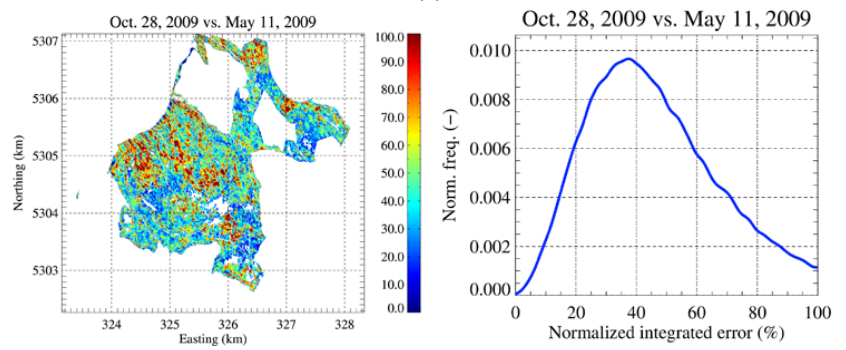

(b)

Fig. 11. Normalized integrated profile difference (NID, \%): maps (UTM coordinates, left) and histogram (right) of (a) wet with respect to dry conditions; (b) spring with respect to autumn.

phase centers $z_{01}$ of the single-layer cells are considered. For $N_{L}=2$, the layers have been distinguished into the ones with lower phase center heights (indicated in the following with $z_{\text {low }}$ ) and the ones with higher phase center heights (indicated in the following with $z_{\text {high }}$ ). Then, the ratio $\chi$ between the power weights associated to $z_{\text {high }}$ and $z_{\text {low }}$ has been calculated. $z_{01}, z_{\text {low }}$ and $z_{\text {high }}$ averagely change from dry to wet conditions mostly within $20 \%$, showing an increase in a great amount of cases. The ratio $\chi$ tends to be higher in wet than in dry conditions (i.e. the scattering contributions close to the canopy top become more dominant). However, the changes are scattered with a large dispersion (up to about 10 $\mathrm{dB}$ ) and are indicating a dielectric-induced ambiguity in the interpretation of the profile amplitudes. No meaningful changes have been observed in the layer widths. These results seem to confirm the hypothesis that the dielectric change induced by a rainfall causes an increase of attenuation without sensitively changing the height locations of the scattering elements. In contrast, the seasonal variations exhibit different
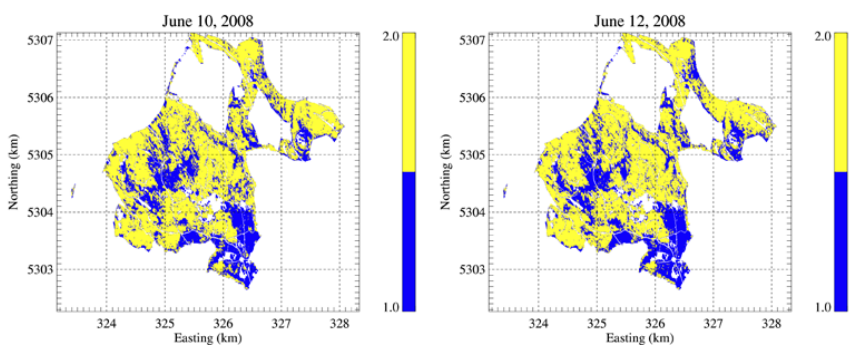

(a)
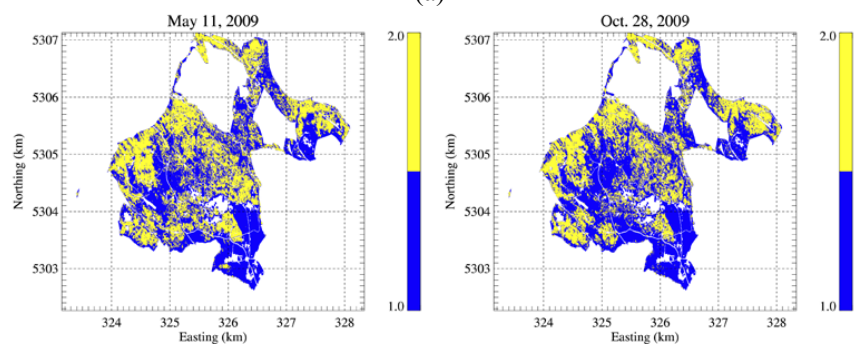

(b)

Fig. 12. Number of fitted volume layers (model order). (a) Dry (left) against wet (right) conditions; (b) spring (left) against autumn (right).

trends. The mean changes of $z_{01}$ in autumn are within $20 \%$ difference with respect to spring, and they are mostly with negative sign, indicating a decrease of height. However, for about $50 \%$ of the cells, $z_{\text {low }}$ is much lower in autumn than in spring. Similarly, $z_{\text {high }}$ generally decreases, although at a lower extent. Conversely, $\chi$ increases from spring to autumn, but less and with a lower dispersion than in the "dry" vs. "wet" case. This suggests that dielectric changes induced by seasonality can increase the attenuation of the volume, but at the same time the relevant scattering elements may appear in different height positions. Layer widths change as well; although without specific spatial patterns, and generally increase from spring to autumn.

\section{RESULTS WITH NON-STATIONARY CONDITIONS}

Up to now the analysis was based on TomoSAR acquisitions performed within few hours, allowing to assume the stationarity of the reflectivity profile within the tomographic set. However, data sets of (realistic) space-borne TomoSAR implementations will build up of images (or interferograms) acquired within weeks or even months. In this case, the stationarity of the reflectivity within the tomographic set will not anymore be guaranteed, as the vertical reflectivity profile may change even from acquisition to acquisition for instance due to rainfalls.

In this perspective, it is of interest to analyze the effects of processing a covariance matrix $\mathbf{R}_{P, I}$ whose elements refer to different "dry" and "wet" acquisitions, and therefore to different reflectivity profiles. To this purpose, the "dry" and "wet" data sets of 2008 have been used. After the interpolation, each data set has the same four uniformly distributed non-zero wavenumbers labelled as "dry" or "wet". A composite covariance matrix has been created with "dry" and "wet" covariances arranged depending on their 

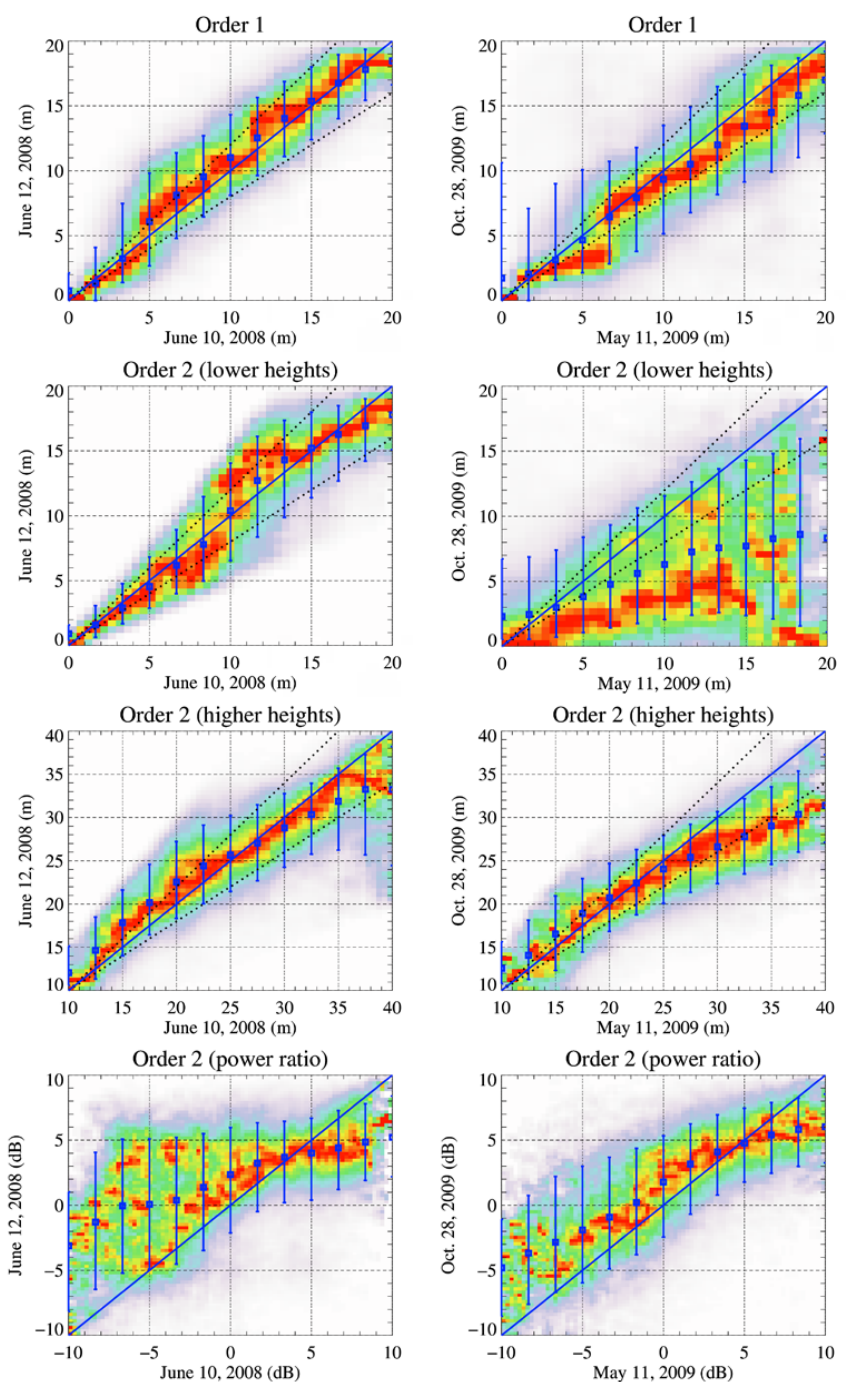

(a)

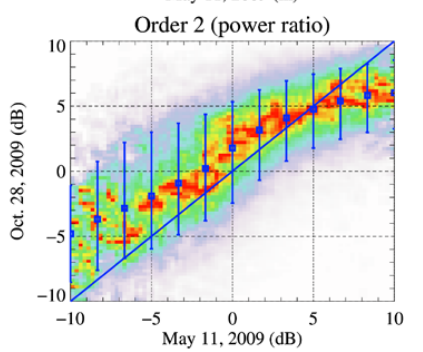

(b)

Fig. 13. From top to bottom: comparison (2-D histograms) of: (1) layer phase center height for model order 1; (2) lower and (3) higher layer phase center heights for model order 2; (4) ratio between the powers of the layers at higher height and at lower heights. The square symbols indicate (conditioned) mean values, while the vertical bars delimit the interval where $75 \%$ of the estimates are found. The dotted lines delimit the $20 \%$ variation interval. Column (a): wet against dry conditions; column (b): autumn against spring.

wavenumber. In this way, in this "synthesized" 12 composite covariance matrices associated to non-stationary dielectric conditions have been created for each cell. The different combinations are listed in Table II.

Fig. 14 shows the Capon HH TomoSAR profiles of the same forest transect as in Fig. 3 obtained from the 12 "mixed" covariance matrices of Table II. When compared to the profiles in dry conditions [left image in Fig. 3(a)], it is apparent that even only one "wet" wavenumber out of four can induce a sensitive change of the profile. This is seen for instance in the relative power contrast between ground and volume. This change of contrast is not homogeneous, but varies from stand to stand. In addition, volume scattering components that were semi-transparent in dry conditions now become well visible. Sharp profiles can become less focused due to the partial inconsistency among covariances. In general,
TABLE II

ANALYZED COMBINATIONS OF CONDITIONS

\begin{tabular}{|c|c|c|}
\hline \multirow{4}{*}{$\begin{array}{l}3 \text { "dry" wavenumbers } \\
1 \text { "wet" wavenumber }\end{array}$} & 1 & $\mathrm{~W}, \mathrm{D}, \mathrm{D}, \mathrm{D}$ \\
\hline & 2 & $\mathrm{D}, \mathrm{W}, \mathrm{D}, \mathrm{D}$ \\
\hline & 3 & $\mathrm{D}, \mathrm{D}, \mathrm{W}, \mathrm{D}$ \\
\hline & 4 & $\mathrm{D}, \mathrm{D}, \mathrm{D}, \mathrm{W}$ \\
\hline \multirow{4}{*}{$\begin{array}{l}2 \text { "dry" wavenumbers } \\
2 \text { "wet" wavenumbers }\end{array}$} & 5 & $\mathrm{~W}, \mathrm{~W}, \mathrm{D}, \mathrm{D}$ \\
\hline & 6 & $\mathrm{D}, \mathrm{W}, \mathrm{W}, \mathrm{D}$ \\
\hline & 7 & $\mathrm{D}, \mathrm{D}, \mathrm{W}, \mathrm{W}$ \\
\hline & 8 & $\mathrm{D}, \mathrm{W}, \mathrm{D}, \mathrm{W}$ \\
\hline \multirow{4}{*}{$\begin{array}{c}1 \text { "dry" wavenumber } \\
3 \text { "wet" wavenumbers }\end{array}$} & 9 & $\mathrm{~W}, \mathrm{~W}, \mathrm{~W}, \mathrm{D}$ \\
\hline & 10 & $\mathrm{D}, \mathrm{W}, \mathrm{W}, \mathrm{W}$ \\
\hline & 11 & $\mathrm{~W}, \mathrm{D}, \mathrm{W}, \mathrm{W}$ \\
\hline & 12 & $\mathrm{~W}, \mathrm{~W}, \mathrm{D}, \mathrm{W}$ \\
\hline
\end{tabular}

"D" stand for "dry" and "W" for "wet". In each set, the (non-zero) wavenumber increases from left to right.

no specific trends can be observed depending if smaller or larger vertical wavenumbers are affected. The height locations of the different scattering components remain widely invariant to the combination.

As a final experiment, the volume-only coherences were estimated for each combination, and used to calculate the $\mathrm{CoM}$ and to separate the scattering layers in the vertical profiles, analogously to what done in Section IV.B and IV.C. The (conditioned) mean values and the $75 \%$ intervals of the estimated parameters with the 12 combinations are compared in Fig. 15 against the homologous quantities obtained from the "dry" data set (horizontal axis). For all parameters, even in the worst case, the change does not practically exceed the change observed using "wet"-only instead of "dry"-only coherences [see Figs 10(a) and 13(a)]. The mean CoM varies within 30\% with respect to the "dry" data set independently of the combination. However, there are cases in which changing one, two, or three wavenumbers to "wet" has the same effect on the $\mathrm{CoM}$, whose variation is well within the $20 \%$ of the "dry"only case.

Concerning now the separated layers, the model order changes only for a very small number of cells (around 5\%) independently of the combination. For $N_{L}=1$, the layer phase centers $z_{01}$ exhibit a variation of less than about $10 \%$ when only one "wet" wavenumber is used. Changes in the mean values are not significant, but a dispersion towards higher values is observed. Both the mean values and the dispersion increase with increasing number of wet wavenumber, although they do not exceed changes of $20 \%$. A similar trend is essentially observable also for $z_{\text {low }}$. Regarding $z_{\text {high }}$, a larger dispersion towards higher values is obtained. In any case, the change of the mean value does not exceed the $30 \%$. While the height positions result to be quite robust to non-stationarities, 

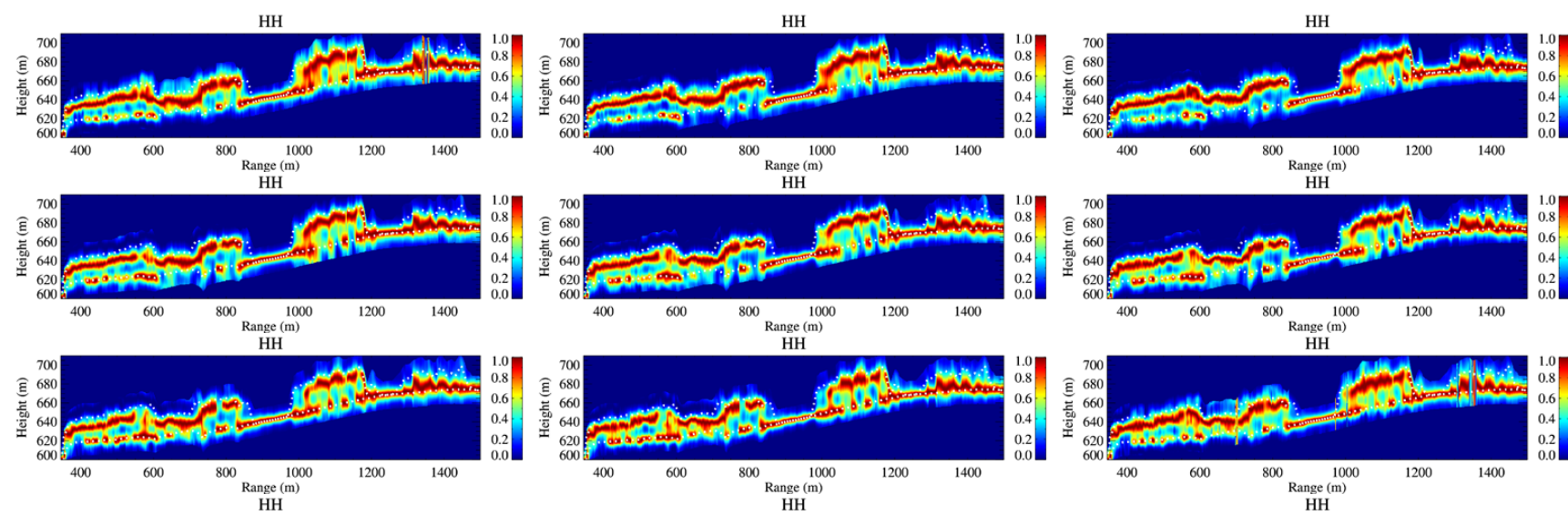

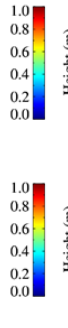

(b)

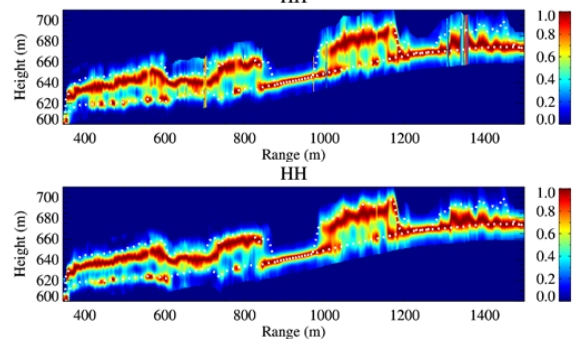

(c)

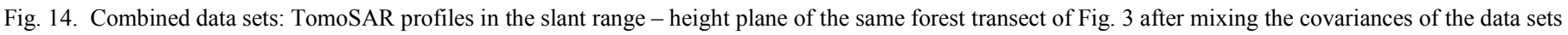

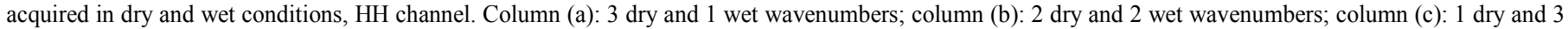
wet wavenumbers. For each column, the profiles are ordered from top to bottom as in Table II. White dotted lines: lidar topography and top height.

the ratio $\chi$ exhibits the largest variation indicating again a certain interpretation ambiguity of the profile amplitudes. A single "wet" wavenumber out of four can change $\chi$ up to 5 $\mathrm{dB}$. This interval increases further if two or three "wet" wavenumbers are used.

\section{DISCUSSION AND CONCLUSIONS}

The impact of dielectric changes due to weather effects as rainfalls and to seasonal variability on vertical reflectivity profiles of forests at L-band has been addressed. The evaluations of the performed experiments clearly demonstrates that, even for a low number of acquisitions (4-5) and a moderate / low vertical resolution (18 - $25 \mathrm{~m})$, dielectric changes appear on the vertical reflectivity profiles. It is expected that with a higher TomoSAR vertical resolution such effects would be reflected over (possibly) a larger number of scattering layers within the same profile.

The experiments indicated that rainfalls essentially increase volume attenuation, as a consequence of both the interception of rain by the tree canopy and the potential rise of the tree water content. In this case, the ground-to-volume ratio can decrease by more than $3 \mathrm{~dB}$ even in mature and structurally complex forest stands, essentially due to a decrease of the ground backscattered power. The expected increase of ground power due to an increase of soil moisture (e.g. water dropped to the soil) is masked out by the higher attenuation. In consequence, the volume-only CoM increases up to $30 \%$. The phase center heights associated to the separated layers remain relatively stable, with a variation between $20 \%$ and $30 \%$. However, the (relative) power of layers close to the canopy top increases with a high dispersion locally up to $10 \mathrm{~dB}$.

Concerning the evaluated seasonal changes, the volume attenuation increases from spring to autumn, as the layers close to the canopy top tend to augment their relative power up to $5 \mathrm{~dB}$. This could be a result of an increase of water content due to a reduction of transpiration. On the other hand, the phase center heights of the scattering layers in general decrease sensitively, possibly indicating a redistribution of water content from twigs and branches to the trunk. Besides, the ground power increases, probably as a consequence of an increase of soil moisture. The reduced transpiration can have played a part in it.

It becomes clear that the estimation of physical forest structure attributes from L-band reflectivity profiles must take into account the ambiguity in interpreting the profile amplitudes. This is a critical issue when it comes to evaluating forest structure dynamics in time, as a dielectric change can be misinterpreted as a structure (i.e. geometric) change. One possibility to face this is to model the dielectric effects and then account or compensate for them in the data inversion process. However, this can be rather challenging even in the case of simple vertical profiles. An alternative is to develop / use algorithms which rely on parameters that are (more) robust against dielectric changes. In this sense, the results suggest that the use of the layer number and relative location might be an option as they appear (more) robust against dielectric changes as in the case of rainfalls. This possibility has already been investigated for the characterization of physical 3-D structure in [31] and [32] across different forest types and conditions.

Dielectric changes occurring in the time required to acquire the tomographic data set affect both repeat-pass and repeated single-pass TomoSAR implementations. In the case of repeatpass implementations, the dielectric changes contribute to a height-dependent temporal decorrelation between acquisitions in each interferometric pair, as addressed in [33] and [34]. In repeated single-pass implementations, even if each interferometric coherence is free of temporal decorrelation, the different coherences refer to different reflectivity profiles as a result of rainfall events or other intra-seasonal dielectric variability. Our experiments have shown that, in the case of 

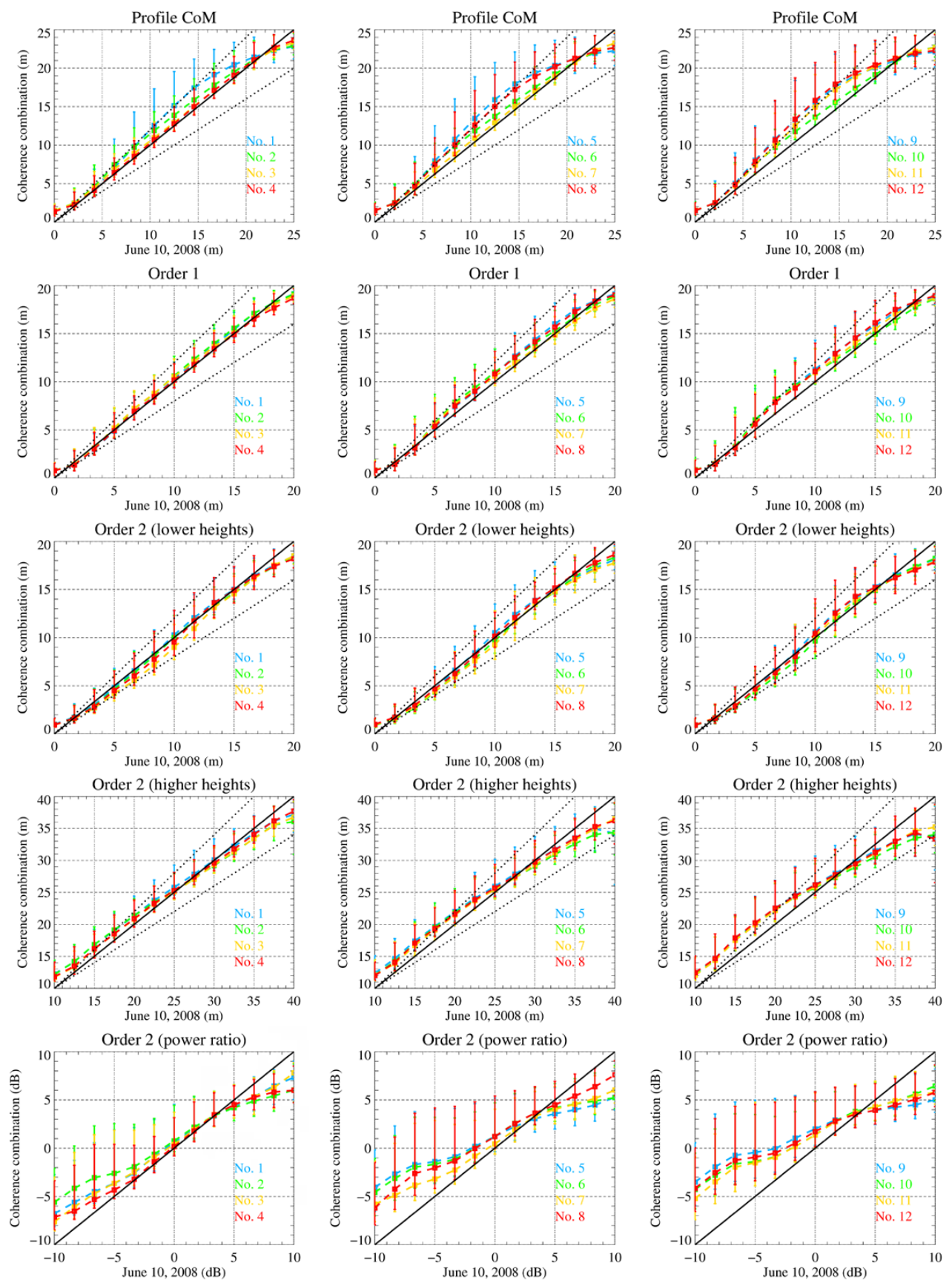

Fig. 15. Analysis of combined data sets. The different combinations are numbered as reported in Table II. From top to bottom: comparisons of: (1) centers of mass of the volume-only profiles; (2) layer phase center height for model order 1; (3) lower and (4) higher layer phase center heights for model order 2; (5) ratio between the powers of the layers at higher height and at lower heights. The square symbols indicate (conditioned) mean values, while the vertical bars delimit the height interval where $75 \%$ of the estimates are found. The dotted black lines delimit the $20 \%$ variation interval. Column (a): combinations $1-4$ ( 3 dry, 1 wet); column (b): combinations 5-8 (2 dry, 2 wet); column (c): combinations 9-12 (1 dry, 3 wet).

rainfall-induced variations of the single-pass interferometric coherence, the relative locations of the estimated vegetation layers can still be reconstructed and vary only within $10 \%$ and $30 \%$. In contrast, the use of the profile amplitudes would again lead to interpretation ambiguities. However, the analysis was limited to just few dryness and wetness conditions for the same forest scenario. On the other hand, intra-seasonal dielectric variations could play a different and non-negligible role [15], whose effect and extent remains to be investigated. Indeed, rather than changing only the volume attenuation, the redistribution of water content may directly affect the visibility of individual scattering elements. In this sense, further investigations are needed to understand to which extent individual changes can be detected from a single polarimetric and / or a single interferometric acquisition. The potential of Differential SAR Tomography, i.e. multi-dimensional SAR imaging [33], [35], to distinguish in such non-stationary scenarios between the spatial (height) and temporal distribution of the backscattered power could be further explored.

Finally, it is worth commenting here that the sensitivity of TomoSAR profiles to dielectric changes is actually a unique 
challenge that remains to be mastered. This opens the door to future applications as for example the quantification of the amount of intercepted rain in forest canopies or monitoring the water status and cycle of a forest and inspire future SAR measurement configurations.

\section{ACKNOWLEDGEMENT}

The authors would like to thank: the E-SAR / F-SAR team for its invaluable effort during the data acquisition and processing; M. Heym, P. Biber (Chair of Forest Growth and Yield) and T. Schneider (Institute of Forest Management) at the Technical University of Munich (TUM) for sharing the field inventory data and the forest type classification map of the Traunstein forest; A. Cantini and S. Marongiu (both from the University of Pisa) for their help in the initial analyses of the data sets during their Master thesis work at DLR.

\section{REFERENCES}

[1] A. Reigber, A. Moreira, "First demonstration of airborne SAR tomography using multibaseline L-band data," IEEE Trans. Geosci. Remote Sens., vol. 38, no. 5, pp. 2142-2152, Sept. 2000.

[2] F. Lombardini, M. Pardini, "Experiments of tomography-based SAR techniques with P-band polarimetric data," Proc. $4^{\text {th }}$ ESA Int. Workshop on Polarimetry and Polarimetric Interferometry, PolInSAR 2009, Frascati, Italy, Jan. 2009

[3] O. Frey, E. Meyer, "Analyzing tomographic SAR data of a forest with respect to frequency, polarization, and focusing techniques," IEEE Trans. Geosci. Remote Sens., vol. 49, no. 10, pp. 3648-3659, Oct. 2009.

[4] S. Tebaldini, F. Rocca, "Multibaseline polarimetric SAR tomography of a boreal forest at P- and L-bands," IEEE Trans. Geosci. Remote Sens., vol. 50, no. 1, pp. 232-246, Jan. 2012.

[5] M. Mariotti d'Alessandro, S. Tebaldini, F. Rocca, "Phenomenology of ground scattering in a tropical forest through polarimetric synthetic aperture radar tomography," IEEE Trans. Geosci. Remote Sens., vol. 51, no. 8, pp. 4430-4437, Aug. 2012.

[6] M. Pardini, J. S. Kim, K. Papathanassiou, I. Hajnsek, "3-D structure observation of African tropical forests with multi-baseline SAR: Results from the AfriSAR campaign," Proc. of 2017 IEEE Int. Geosci. Remote Sens. Symp., IGARSS 2017, Fort Worth, TX, USA, pp. 4288-4291, Jul. 2017.

[7] A. Hamadi, P. Borderies, C. Albinet, T. Koleck, L. Villard, D. H. T. Minh, T. Le Toan, B. Burban, "Temporal coherence of tropical forests at P-band: Dry and rainy season," IEEE Geosci. Remote Sens. Lett., vol. 12, no. 3, pp. 557-561, Mar. 2015.

[8] L. M. H. Ulander, A. Monteith, "Time series of P- and L-band forest backscatter from BorealScat," Proc. of 2017 IEEE Int. Geosci. Remote Sens. Symp., IGARSS 2017, Fort Worth, TX, USA, pp. 4310-4313, Jul. 2017.

[9] M. Watanabe, T. Motohka, T. Shiraishi, R. Bahadur Thapa, C. Yonezawa, K. Nakamura, M Shinaba, "Multitemporal fluctuations of Lband backscatter from a Japanese forest," IEEE Trans. Geosci. Remote Sens., vol. 53, no. 11, pp. 5799-5813, Nov. 2015.

[10] J. De Jong, W. Klaassen, A. Ballast, "Rain storage in forests detected with ERS tandem mission SAR," Remote Sensing of Environment, vol. 72, issue 2, pp. 170-180, 2000.

[11] F. Kugler, D. Schulze, I. Hajnsek, H. Pretzsch, K. Papathanassiou, "TanDEM-X Pol-InSAR performance for forest height estimation," IEEE Trans. Geosci. Remote Sens., vol. 52, no. 10, pp. 6404-6422, Oct. 2014.

[12] M. J. Soja, J. I. H. Askne, L. M. H. Ulander, "Estimation of boreal forest properties from TanDEM-X using inversion of the water cloud model," IEEE Geosci. Remote Sens. Lett., vol. 17, no. 7, pp. 997-1001, Jul. 2017.

[13] F. De Zan, M. Zonno, P. Lopez-Dekker, "Phase inconsistencies and multiple scattering in SAR interferometry," IEEE Trans. Geosci. Remote Sens., vol. 53, no. 12, pp. 6608-6616, Dec. 2015.

[14] M. Pardini, A. Cantini, F. Lombardini, K. Papathanassiou, "3-D structure of forests: First analysis of tomogram changes due to weather and seasonal effects at L-band," Proc. $10^{\text {th }}$ European Conf. Synthetic Aperture Radar, Berlin, Germany, pp. 48-51, Jun. 2014.
[15] D. M. Gates, "Water relations of forest trees," IEEE Trans. Geosci. Remote Sens., vol. 29, no. 6, pp. 836-842, Nov. 1991.

[16] A. Wallner, A. Elatawneh. T. Schneider, T. Knoke, "Estimation of forest structural information using RapidEye satellite data," Forestry: An International Journal of Forest Research, vol. 88, issue 1, pp. 96-107, Jan. 2015.

[17] S. K. Lee, F. Kugler, K. Papathanassiou, I. Hajnsek, "Quantification of temporal decorrelation effects at L-band for polarimetric SAR interferometry applications," IEEE Journal Sel. Topics Applied Earth Obs. Remote Sens., vol. 6, no. 3, pp. 1351-1367, Mar. 2013.

[18] M. Pardini, A. Toraño Caicoya, F. Kugler, K. Papathanassiou, "On the estimation of forest vertical structure from multibaseline polarimetric SAR," Proc. of 2012 IEEE Int. Geosci. Remote Sens. Symp., IGARSS 2012, Munich, Germany, pp. 3443-3446, Jul. 2012.

[19] K. Papathanassiou, S. Cloude, "Single-baseline polarimetric SAR interferometry," IEEE Trans. Geosci. Remote Sens., vol. 39, no. 11, pp. 2352-2363, Nov. 2001.

[20] Y. Huang, L. Ferro-Famil, F. Lombardini, "Improved tomographic SAR focusing using automatic baseline error compensation," Proc. $5^{\text {th }}$ ESA Int. Workshop on Polarimetry and Polarimetric Interferometry, PolInSAR 2011, Frascati, Italy, Jan. 2011.

[21] M. Pardini, K. Papathanassiou, "A two-step phase calibration method for tomographic application with airborne SAR data," Proc. $10^{\text {th }}$ European Conf. Synthetic Aperture Radar, Berlin, Germany, Jun. 2014.

[22] F. Lombardini, M. Pardini, "3-D SAR tomography: The multibaseline sector interpolation approach," IEEE Geosci. Remote Sens. Lett., vol. 5, no. 4, pp. 630-634, Oct. 2008.

[23] F. Bordoni, A. Jakobsson, F. Lombardini, F. Gini, "Multibaseline crosstrack SAR interferometry using interpolated arrays," IEEE Trans. Aerospace Electr. Syst., vol. 41, no 4, pp. 1473-1482, Oct. 2005.

[24] F. Lombardini, M. Pardini, L. Verrazzani, "A robust multibaseline sector interpolator for SAR tomography," Proc. $7^{\text {th }}$ European Conf. Synthetic Aperture Radar, Friedrichshafen, Germany, Jun. 2008.

[25] M. Pardini, K. Papathanassiou, "On the estimation of ground and volume polarimetric covariances in forest scenarios with SAR tomography," IEEE Geosci. Remote Sens. Lett., vol. 14, no. 10, pp. 1860-1864, Oct. 2017.

[26] S. Cloude, K. Papathanassiou, "Three-stage inversion process for polarimetric SAR interferometry," IEE Proc. - Radar Sonar Navig., vol. 150, no. 3, 125- 134, Jun. 2003.

[27] F. Gini, F. Lombardini, M. Montanari, "Layover solution in multibaseline SAR interferometry," IEEE Trans. Aerospace Electr. Syst., vol. 38, no 4, pp. 1344-1356, Oct. 2002.

[28] B. Ottersten, P. Stoica, R. Roy, "Covariance matching estimation techniques for array signal processing applications," Digital Sign. Proc., vol. 8, no. 3, pp. 185-210, Jul. 1998.

[29] P. Stoica, O. Besson, A. B. Gershman, "Direction-of-arrival estimation of an amplitude distorted wavefront," IEEE Trans. Sig. Proc., vol. 49, no. 2, pp. 269-276, Feb. 2001.

[30] F. Lombardini, J. Ender, L. Rößing, M. Galletto, L. Verrazzani, "Experiments of interferometric layover solution with the three-antenna airborne AER-II SAR system," Proc. of 2004 IEEE Int. Geosci. Remote Sens. Symp., IGARSS 2004, Anchorage, Alaska, Sept. 2004.

[31] M. Tello, V. Cazcarra Bes, K. Papathanassiou, "Assessment of forest structure estimation by means of SAR tomography: Potential and limitations," Proc. of 2016 IEEE Int. Geosci. Remote Sens. Symp., IGARSS 2016, Beijing, China, pp. 32-35, Jul. 2016.

[32] V. Cazcarra-Bes, M. Tello, R. Fischer, M. Heym, K. Papathanassiou, "Monitoring of forest structure dynamics by means of L-band SAR tomography, Remote Sensing, vol. 9, no. 12, 2017.

[33] F. Lombardini, F. Cai, F. Viviani, D. Pasculli, "Multidimensional SAR tomography for complex non-stationary scenes: COSMO-SkyMed urban and P-band forest results," Proc. of 2012 IEEE Int. Geosci. Remote Sens. Symp., IGARSS 2012, Munich, Germany, July 2012.

[34] M. Lavalle, S. Hensley, "Extraction of structural and dynamic properties of forests from polarimetric-interferometric SAR data affected by temporal decorrelation," IEEE Trans. Geosci. Remote Sens., vol. 53, no. 9, pp. 4752-4767, Sept. 2015.

[35] F. Lombardini, F. Cai, "Temporal decorrelation-robust SAR tomography," IEEE Trans. Geosci. Remote Sens., vol. 52, no. 9, pp. 5412-5421, Sept. 2014. 


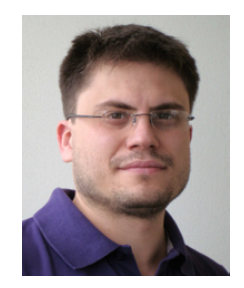

Matteo Pardini (S'02 - M'10) received the Ms. Eng. Degree (cum laude) in Telecommunication Engineering and the Ph.D. in Information Engineering from the University of Pisa (Italy) in 2006 and 2010, respectively.

In January 2010, he joined the Radar Concepts department of the Microwaves and Radar Institute of the German Aerospace Center (DLR) in Oberpfaffenhofen (Germany) as a research scientist, after a visiting research period from August to December 2009. In 2017, he was Visiting Scientist at the Department of Geographical Sciences of the University of Maryland (USA). His main research interests are in Synthetic Aperture Radar (SAR) tomographic and polarimetric interferometric processing for 3-D bio/geophysical information extraction over natural volumes (forest, agriculture, ice), as well as in SAR mission design and SAR mission performance analysis.

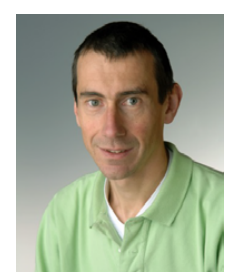

Konstantinos P. Papathanassiou (M'02, SM'03, FM'14) received the Dipl. Ing degree (Honors) in 1994 and the Dr. degree (Honors) in 1999 from the Technical University of Graz, Austria. From 1992 to 1994 he was with the Institute for Digital Image Processing (DIBAG) of Joanneum Research, in Graz, Austria. Between 1995 and 1999 he worked at the Microwaves and Radar Institute (HR) of the German Aerospace Center (DLR), in Oberpfaffenhofen, Germany. From 1999 to 2000 he was an EU post-doctoral fellow with Applied Electromagnetics (AEL) in St. Andrews, Scotland. Since October 2000 he is again with the Microwaves and Radar Institute (HR) of the German Aerospace Center (DLR). Actually he is a senior scientist leading the Information Retrieval research group at DLR-HR.

His main research interests are in polarimetric and interferometric processing and calibration techniques, polarimetric SAR interferometry, and the quantitative parameter estimation from SAR data, as well as in SAR mission design and SAR mission performance analysis. He is member of DLR's TanDEM-X and Tandem-L science teams, JAXA's ALOS-2 Cal-Val teams, ESA's BIOMASS Mission Advisory Group, SAOCOM-SC Expert Team, and JAXA's Carbon and Kyoto Initiative and NASA's GEDI Mission Science Team. He was awarded with the IEEE GRSS IGARSS Symposium Prize Paper Award in 1998, the Best Paper Award of the European SAR Conference (EUSAR) in 2002 and the DLR science award in 2002. In 2011 he was awarded with DLR's Senior Scientist Award.

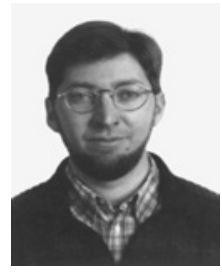

Fabrizio Lombardini (SM'03) received the Italian Laurea degree (with honors) in electronic engineering and the $\mathrm{PhD}$ degree in telecommunication engineering from the University of Pisa, Italy, in 1993 and 1997, respectively. He was then granted by the EU a Marie Curie Fellowship of the Training and Mobility of Researchers (TMR) Program, which he spent as Postdoctoral Researcher at the Department of
Electronic and Electrical Engineering of University College London, U.K., from 1998 to 1999. Then, he joined the Department of Information Engineering of University of Pisa, where he currently holds the position of Associate Professor in Telecommunications.

He has given lectures at universities and institutions in Italy and abroad, and has organized and chaired more than 20 special sessions on Synthetic Aperture Radar (SAR) multibaseline/multichannel interferometry, coherent data combination, and three-/multi-dimensional techniques at international conferences; he has served as Editor of the EURASIP Journal of Applied Signal Processing (JASP) special issue on "Advances in Interferometric SAR Processing", is Associate Editor of the IEEE Journal of Selected Topics in Applied Earth Observations and Remote Sensing (JSTARS), and has been lecturer or co-lecturer of 10 tutorials on SAR tomography and multidimensional SAR imaging.

Dr. Lombardini's general research interests are in the areas of statistical signal processing, estimation and detection, super-resolution spectral analysis, array processing, irregular sampling, and performance bounds evaluation, with application to radar systems and SAR environmental remote sensing. He has authored or co-authored more than 140 papers (peer-review journals and proceedings of international conferences) on these subjects. In particular, his research interests include multibaseline and multifrequency interferometric SAR algorithms and systems, both cross- and along-track, 3D SAR tomography, and differential SAR interferometry. He has originated the new mode of 4D SAR imaging (differential SAR tomography), and has recently engaged research on coherent localization in radio frequency identification (RFID) systems. $\mathrm{He}$ has participated to various international and national research and industry oriented projects. 